\title{
La participación política indígena y las organizaciones de la sociedad civil. Análisis de la zona metropolitana de Monterrey, México*
}

\author{
Indigenous political participation and civil society \\ organizations. Analysis of the metropolitan area of Monterrey, \\ Mexico
}

A participação política indígena e as organizações da sociedade civil. Análise da região metropolitana de Monterrey, México

\author{
José Fredman Mendoza Ibarra**, \\ Kate del Rosario Rodríguez Alejandro***
}

\begin{abstract}
RESUMEN
La migración indígena a ciudades industriales como la zona me-

tropolitana de Monterrey (ZMM) en el Estado de Nuevo León, al noreste de México, ha provocado la reconfiguración jurídica y política de la entidad mediante la implementación de procesos de consulta previa, libre e informada a personas y pueblos indígenas. En ese sentido, la capacidad de reacción por parte de los gobiernos locales y los diversos actores involucrados, como las organizaciones de la sociedad civil (OSC) indígenas y proindíge-
\end{abstract}

Palabras clave: consulta previa,

derechos

indígenas,

migración

indígena, OSC

indígenas y

proindígenas,

participación

política indígena.

\footnotetext{
* El presente documento es fruto del trabajo de campo realizado en el marco de la tesis doctoral Participación político-electoral de personas indígenas en ciudades industriales. Análisis de Monterrey y su Área Metropolitana en la Universidad Autónoma de Nuevo León, México, con disertación en 2019 y financiada por el Consejo Nacional de Ciencia y Tecnología (CONACYT) del gobierno de México.

** Doctor en Ciencias Políticas por la Universidad Autónoma de Nuevo León (UANL). Actualmente es profesor investigador de la Universidad de Monterrey (UDEM) y miembro del Sistema Nacional de Investigadores del Consejo Nacional de Ciencia y Tecnología (CONACYT) en México. E-mail: jose.mendozai@udem.edu

*** Estudiante de Relaciones Internacionales en la Universdad Autónoma de Nuevo León (UANL), Monterrey, México. Asistente de investigación en proyecto CONACYT. E-mail: kate.rdz99@gmail.com
} 
nas, en la promoción, garantía y respeto de los derechos indígenas de participación política ha sido variada. A partir de la herramienta cualitativa de entrevista semiestandarizada, en 2018 se reconoció el papel que tienen tres de las principales OSC de Nuevo León en los procesos de consulta previa y su capacidad de vinculación y materialización de derechos de participación política indígena. Se puede observar que, a pesar del cumplimiento en términos formales y procedimentales de la consulta indígena, los esfuerzos de las OSC no han podido materializarse en una mayor participación y representación política indígena en Nuevo León debido a la desconfianza y débil sinergia entre las asociaciones.

\begin{abstract}
Indigenous migration to industrial cities such as Monterrey's metropolitan area (ZMM) in the State of Nuevo León in northeastern Mexico has led to its legal and political reconfiguration processes of free, prior, and informed consultation with indigenous individuals and peoples. In this sense, the capacity of local governments and the various actors involved, such as indigenous and pro-indigenous civil society organizations (CSOs), to react in promoting, guaranteeing, and respecting indigenous rights to political participation has been varied. Based on the qualitative tool of semi-standardized interviews, in 2018, the role of three of the principal CSOs in Nuevo León in the processes of prior consultation and their capacity to link and materialize indigenous political participation rights was recognized. We observe that despite compliance in formal and procedural terms with the indigenous consultation, the efforts of CSOs have not been able to materialize in greater participation and indigenous political representation in Nuevo León due to mistrust and weak synergy among the associations.
\end{abstract}

\section{RESUMO}

A migração indígena para cidades industriais como a região metropolitana de Monterrey (RMM) no Estado de Nuevo León, no nordeste do México, levou à reconfiguração jurídica e política da entidade através da implementação de processos de consulta prévia, livre e informada às pessoas e aos povos indígenas. A esse respeito, a capacidade de reação dos governos locais e dos diversos atores envolvidos, como as organizações da sociedade civil (OSC) indígenas e de apoio aos povos indígenas, na promoção, na garantia e no respeito dos direitos indígenas à participação política tem sido variada. A partir da ferramenta qualitativa de
Keywords: prior consultation, indigenous rights, indigenous migration, indigenous and pro-indigenous CSOs, indigenous political participation.

Palavras-chave: consulta prévia, direitos indígenas, migração indígena, OSC indígenas e de apoio aos povos indígenas, participação política indígena. 
entrevistas semipadronizadas, em 2018 foi reconhecido o papel de três das principais OSC de Nuevo León nos processos de consulta prévia e sua capacidade de vinculação e materialização dos direitos de participação política indígena. É possível observar que, apesar do cumprimento em termos formais e processuais da consulta indígena, os esforços das OSC não puderam se materializar em uma maior participação e representação política indígena em Nuevo León devido à desconfiança e à fraca sinergia entre as associações. 


\section{Introducción}

La exclusión del paradigma político de las minorías étnicas en América Latina provocó, en la segunda mitad del siglo XX, la irrupción de múltiples movimientos y organizaciones indígenas en la arena política de los estados-nación. Con ello, lo anterior adquirió relevancia debido a que ponían en jaque la forma de operar de los gobiernos y exigían, de manera real y precisa, respuestas institucionales para el reconocimiento de sus derechos políticos y la satisfacción de sus demandas mediante mecanismos democráticos (Van Cott, 1995). Es decir, lo que se estaba formando en aquel entonces era el comienzo de un diálogo entre iguales, pero con la exigencia del reconocimiento de las diferencias culturales, siendo Ecuador, Bolivia y Perú los países más representativos de estos procesos (Martí, 2013).

Para el caso de México, la emancipación política del mestizo no ha concomitado con la emancipación política de las personas indígenas (Mendoza, 2019). Con ello desemboca una falsa representación de lo indígena en medios de comunicación (Marañón, Saldierna \& Muñiz, 2013) y una constante visión de paternalismo, autoritarismo y desigualdad estructural al momento de implementar políticas gubernamentales para el desarrollo de los pueblos indígenas en el país (González Casanova, 2013; Monsiváis, 2014). Además, las irrupciones de movimientos políticos de corte indígena coincidieron con la amplia migración de zonas rurales hacia ciudades industriales a finales del siglo XX (Granados, 2005).

La zona metropolitana de Monterrey (ZMM) ha pasado de tener poco más de 80.000 habitantes indígenas en 2010 a más de 350.000 habitantes indígenas en 2015, de acuerdo con el Instituto Nacional de Estadística, Geografía e Informática (INEGI). Con ello, la participación política, concebida como el derecho que tienen las personas y grupos a incidir en la toma de decisiones en la esfera pública y que además representa el eje rector de las democracias modernas (Cohen \& Arato 1992), tendrá también que adaptarse a la realidad etnocultural que traen consigo los flujos migratorios en las entidades receptoras (Assies, 2009).

Debido a la diversidad étnica en Nuevo León, es la migración indígena proveniente de los estados de San Luis Potosí, Veracruz y el estado de México, y que son hablantes de lenguas como el náhuatl, el 
huasteco, el otomí, el zapoteco y el mixteco, la que mayor presencia tiene en la entidad. Se ha reconocido que los principales motivos de migración hacia la ZMM radican en una mayor percepción de oportunidades laborales y educativas en Nuevo León, al tiempo de que se hace visible que una vez asentadas las comunidades indígenas en el estado, resulta difícil la reproducción de formas de organización política y de vida comunitaria tradicionales (Cerda \& Gallegos, 2008; Wright \& Aguirre, 2018). Por ejemplo, Mendoza, Martínez y Rodríguez (2020) han señalado que las reconfiguraciones del papel de las mujeres indígenas en un contexto migratorio en la ZMM y en la zona metropolitana de Guadalajara (ZMG) significaron: 1) cambios en el rol de la mujer dentro y fuera de la comunidad, 2) cambios en el acceso a ejercicio de derechos debido a un mayor acceso a la educación y 3) la oportunidad de disminuir la discriminación mediante la dignificación del trabajo y la apropiación de espacios públicos.

De esta manera, los actores involucrados en esta nueva realidad etnocultural la entienden como una realidad que permite, desde lo local, el ejercicio de ciudadanía culturalmente diferenciada, pero con la capacidad de que en el contexto local se adquieran capacidades interculturales para la defensa, promoción y ejercicio de los derechos indígenas (Cairo \& Rozo, 2006; Martínez, 2015), como son las organizaciones de la sociedad civil (OSC), los partidos políticos, las empresas, legisladores(as), universidades y gobierno quienes tienen la firme responsabilidad de cristalizar los derechos de las personas indígenas en políticas más participativas y congruentes con lo establecido en las leyes y convenios internacionales (Muñoz, 2014). Entre estos derechos indígenas, están los concernientes al derecho de participación política y que encuentra una posibilidad de materialización en el derecho a la consulta previa, libre e informada. Este derecho, dicho sea de paso, está consagrado en la Constitución política de los Estados Unidos Mexicanos (CPEUM) desde 2001, en el Convenio 169 de la Organización Internacional del Trabajo (OIT) (C169) firmado por México en 1990, en la Declaración de la Naciones Unidas sobre los Derechos de los Pueblos Indígenas en 2007 y en la Ley de los Derechos de las Personas Indígenas y Afromexicanas en Nuevo León en 2012.

Resulta conveniente matizar algunas pautas interpretativas que servirán para una mayor comprensión del presente artículo. Primero, 
la conformación histórica de las organizaciones de la sociedad civil en Nuevo León estuvo incentivada por el déficit de acciones gubernamentales y de las principales instituciones políticas en la entidad, como lo fueron los partidos políticos. Segundo, debido a que la ZMM no cuenta con comunidades indígenas originarias, los tres tipos de migración y asentamiento identificados en la entidad fueron aislados, dispersos y congregados ${ }^{4}$ (Durin, 2003), lo que provocó la aparición de dos tipos de organizaciones civiles: indígenas y proindígenas, pues la ausencia del Estado motivó de múltiples maneras la participación de las personas indígenas y no indígenas en el reconocimiento de los problemas que padecían las personas indígenas en los tres tipos de asentamientos, sobre todo los congregados ${ }^{5}$. Tercero, para el caso de las organizaciones civiles indígenas, son estas en su mayoría las que fueron fundadas y son lideradas por personas indígenas relacionadas con el Consejo de Desarrollo Social de los centros interculturales de la Secretaría de Desarrollo Social (SEDESOL), como el caso de Nuevo León Árbol, fundada en 2008, y Zihuakali, como una iniciativa de Zihuame y que luego pasó a formar parte de las casas de la mujer de la Comisión Nacional para el Desarrollo de los Pueblos Indígenas (CDI) (García, 2013) ${ }^{6}$.

4 Para la tipología de migración/asentamiento aislado, se tiene en cuenta que las personas indígenas viven en el mismo lugar de trabajo; para el caso del asentamiento disperso, las personas indígenas viven de forma dispersa en la ciudad, sin generar comunidades grandes y representativas en término numéricos; y, para el tipo congregado, viven al menos 20 familias con el mismo lugar de origen en la misma área residencial.

5 Por ejemplo, la primera organización civil que trabajó a favor de las personas indígenas fue Zihuame Mochilla en 2003, al tiempo en que se instaló en Monterrey la oficina de atención de la Comisión Nacional para el Desarrollo de los Pueblos Indígenas (CDI) que pertenecía al gobierno federal. Zihuame tiene la particularidad de ser una asociación liderada por mujeres no indígenas que trabajan de la mano con la comunidad para el mejoramiento de sus condiciones de vida en la ciudad y el respeto y cumplimiento de sus derechos. Cabe señalar que los primeros trabajos de Zihuame Mochilla se remontan al 2001 y estuvieron relacionados con los procesos de reubicación y tenencia de la tierra de la comunidad nahua que habitaba en el lecho del río La Silla (zona que comprende un río que cruza los municipios metropolitanos de Santa Catarina, Monterrey, Guadalupe y Juárez, y que históricamente es susceptible a grandes inundaciones).

6 Cabe sañalar que la tipología de la OSC indígena y proindígena desarrollada por García (2013), donde describe a las asociaciones indígenas y grupos proindígenas, cambia para efectos empíricos y analíticos en el presente trabajo. Aquí tomamos en cuenta la relevancia que las asociaciones, para nosotros indígenas (específicamente Nuevo León Árbol de Todas las Raíces y Zihuakali Casa de la Mujer Indígena), pero para Tello proindígenas, han tenido en los últimos procesos de consulta, como en los realizados en 2011 para la Ley de Derechos de Personas Indígenas en Nuevo León, en 2016 para el Plan Estatal de Desarrollo y la consulta de 2020 realizada por la Comisión Estatal Electoral 
En ese sentido, el presente artículo tiene como finalidad estudiar el papel de las organizaciones civiles indígenas y proindígenas en la materialización del derecho de participación política a través de la consulta indígena y se tiene como hipótesis que la proximidad a las OSC indígenas y proindígenas influye en la participación política indígena en Nuevo León. Para comprobar lo anterior, se realizaron entrevistas semiestandarizadas a tres OSC indígenas y proindígenas a las que se tuvo acceso: Zihuame Mochilla, Zihuakali Casa de la Mujer Indígena y Nuevo León Árbol de Todas las Raíces.

El artículo se divide en cuatro secciones. La primera establece la descripción de la consulta previa, libre e informada como derecho en México mediante la descripción del Convenio 169 de la Organización Internacional del Trabajo. La segunda sección brinda algunas pautas interpretativas de corte teórico que nos permitirán comprender la importancia de la participación política, así como la descripción de procesos de participación indígena en Nuevo León. En la tercera sección se realiza el análisis empleando diversas técnicas de investigación cualitativas, como entrevistas semiestandarizadas a informantes clave, así como la utilización del software NVivo 12 para el tratamiento de datos. Finalmente, como cuarta sección, se esbozan algunas reflexiones finales acerca de los principales hallazgos del estudio y sus implicaciones en la participación política indígena en general.

\section{La consulta previa, libre e informada como derecho en México}

El Convenio 169 de la Organización Internacional del Trabajo (OIT) constituye una pieza fundamental para el ejercicio del derecho a la participación política de las personas indígenas en México. El compromiso que adquieren los estados con las personas indígenas tiene dos postulados básicos: 1) el derecho a preservar y fortalecer la cultura, instituciones y visiones de vida y 2) el derecho a la participación activa y efectiva en las decisiones públicas y privadas que les afectan (OIT, 2014). En ese sentido, uno de los mecanismos que otorga el Convenio 169 es el estipulado a partir del derecho a la participación por medio

Nuevo León, en relación con la participación y representación política indígena en el Congreso y los ayuntamientos. 
de la consulta previa y que implica, invariablemente, el contacto entre las autoridades gubernamentales y las personas y comunidades indígenas en consciente ejercicio de libre autodeterminación como pueblo (Rea Granados, 2015; Wright, 2018).

En México, el debate en torno a la autonomía responde a la existencia de por lo menos dos formas distintas de concebir a la democracia y a la ciudadanía (Tamayo, 2006). La ciudadanía indígena se entiende como el ejercicio de derechos y obligaciones a partir de tres elementos fundamentales: los derechos, las prácticas y la identidad; estos permiten relacionarse ya sea en igualdad de circunstancias o de manera desigual con distintos actores políticos (Martínez, 2012; Ochman, 2006). Es decir, el ejercicio de ciudadanía indígena "permite reconocer que no solamente es el Estado el que históricamente ha socavado derechos de las personas y pueblos indígenas, sino también diversos actores sociales han establecido relaciones de poder desiguales y verticales" (Mendoza, Martínez \& Rodríguez, 2020, p. 62) en detrimento de las personas y los pueblos indígenas.

Por un lado, concebir el cúmulo de derechos y obligaciones que conlleva la ciudadanía en términos liberales o étnicos y, por otro lado, la forma de interpretar la manera cómo hacia el interior del territorio mexicano las prácticas democráticas son el resultado del diálogo entre el sistema político convencional de la democracia representativa y el sistema de usos y costumbres de las comunidades indígenas como forma de gobierno y de participación hacia el interior de las comunidades mediante la tradición (1) participativa, (2) de carácter colectivo y comunal donde (3) los principios de honestidad y solidaridad priman en principio por sobre los intereses individuales (Melgar, 1991).

Es conveniente señalar que estas diversas formas de concebir la autonomía indígena como un derecho político y, en suma, democrático, advierte la comprensión de la 1) autoafirmación, 2) la autodefinición, 3) la autodelimitación y 4) la autodisposición interna y externa de las personas y pueblos indígenas (López, 2006). De acuerdo con Obdieta Chalbaud (1993) y López Bárcenas (2006), el derecho de autoafirmación radica en la capacidad que tiene un pueblo para proclamarse existente mediante el derecho de autodefinición que permite a un pueblo determinar quiénes son las personas que lo conforman a través del derecho de autodelimitación para la determinación de su territorio y con 
completo ejercicio de autodisposición como derecho a organizarse y darse su propio gobierno.

Además de lo anterior, las reivindicaciones políticas de las personas y comunidades indígenas están siempre en constante diálogo de manera externa con el sistema político y normas vigentes, y de manera interna, en constante lucha contra alguna de las formas de organización hacia el interior de la comunidad, sus tradiciones y normas (Kymlicka, 1996; López, 2006). Es decir, el cambio es constante, el diálogo permanente y las reconfiguraciones políticas responden a dos visiones del mundo distintas y, en ocasiones, excluyentes entre sí.

Ahora bien, en términos de derechos políticos de autonomía y de participación política, es importante tomar en cuenta que el ejercicio de este tipo de derechos de personas y comunidades indígenas comprende, principalmente, y en sintonía con Kymlicka (1996), dos dimensiones: la dimensión interna y la dimensión externa. La primera de ellas está relacionada con la autonomía y el gobierno hacia el interior de las comunidades, mientras que la segunda, la dimensión externa, tiene que ver con la participación y el diálogo a través de los mecanismos institucionales y sobre temas relacionados con el Estado al que pertenecen y, por lo tanto, con el sistema político vigente (Tomaselli, 2016).

Asimismo, en México -como Estado que ha ratificado el Convenio 169 en 1990 de la OIT, que además este último se encuentra reforzado con la Declaración de Naciones Unidas sobre los Derechos de los Pueblos Indígenas (Naciones Unidas, 2007)- desde 1992 y mediante reforma constitucional, se reconoce por primera vez su carácter pluricultural en la Constitución política de los Estados Unidos Mexicanos (CPEUM) y para 2001, con la reforma constitucional del artículo $2^{\circ}$, se dio paso al reconocimiento y obligatoriedad de garantizar derechos colectivos, como la libre determinación, autonomía, participación y representación política por parte de la federación, los estados y los municipios (Mendoza, 2019; Wright \& Aguirre, 2018). Cabe destacar que en los estados de América Latina, la adopción del Convenio 169, con excepción de El Salvador, Panamá y Uruguay, fue también fruto de movilizaciones estratégicas y del efecto boomerang (Keck \& Sikkink, 1998) de los movimientos y protestas indígenas para reivindicar sus derechos tanto individuales como colectivos (Wright \& Aguirre, 2018). 
El Convenio 169, en su artículo $6^{\circ}$, estipula claramente que los gobiernos deberán 1) consultar a los pueblos indígenas a través de procedimientos apropiados, 2) establecer los medios para que los pueblos indígenas participen y 3) establecer los medios para que las instituciones gubernamentales atienden de manera integral las iniciativas de los pueblos indígenas (2014); todo en lo concerniente a las medidas legislativas, administrativas, políticas y económicas susceptibles a afectarles directa o indirectamente con la finalidad de lograr el consenso de las medidas propuestas y de reconocer la participación política efectiva de las personas y pueblos indígenas (Wright \& Aguirre, 2018).

De este modo, el derecho de la consulta en México, además de tener su base en la Constitución, convenios internacionales y declaraciones universales, significó la reconfiguración de las relaciones entre el Estado y los pueblos indígenas (Juan-Martínez, 2019) debido a que la materialización de los derechos indígenas recae, principalmente, en los gobierno locales y en los instrumentos de gobernanza, transparencia, rendición de cuentas y de participación ciudadana promovidos por las instituciones gubernamentales, partidos políticos y demás actores de la sociedad civil, como lo son las asociaciones civiles indígenas, proindígenas y las universidades. Sin embargo, ¿cómo se implementa la consulta indígena en un contexto migratorio?

\section{La participación política indígena. Pautas interpretativas}

En América Latina durante varios siglos se ha concebido al progreso y al desarrollo como un rechazo al pasado y a las personas y culturas descendientes de los antiguos pueblos indígenas asentados antes de la conquista española. De acuerdo con Mendoza (2019), esclarecer los múltiples factores que influyen en la participación política indígena en México, y en América Latina en general, precisa de esfuerzos teóricos y empíricos que diluciden y ayuden a comprender las causas, efectos, obstáculos y alcances de la participación indígena.

No obstante, los procesos de participación política indígena se pueden reconocer a partir de tres etapas: 1) la irrupción de movimientos sociales indígenas, 2) la crisis de los estados-naciones y 3) los procesos interculturales y de pluralismo jurídico que refuerza el intercambio y el diálogo entre los actores estatales y los pueblos indígenas (Martí, 
2013; Mendoza, 2019; Tresierra, 1995). Cabe destacar que la profusa estigmatización a la que está sujeta la población indígena impacta, invariablemente, en su capacidad de ejercer derechos políticos como los de participación y representación política (Pajuelo, 2006; Domínguez \& Santiago, 2014).

Por participación política indígena entendemos como aquella participación, que es la base de la democracia misma, que busca la representación y el reconocimiento de la diversidad en términos identitarios:

La podemos situar en espacios categóricos de ciudadanía, así como en lo relacionado con estructuras de poder y con el ejercicio de éste en las instituciones del Estado, en los sistemas de gobiernos propios de cada comunidad o mediante organizaciones de la sociedad civil que promueven y/o canalizan las demandas de determinada comunidad indígena para ser incorporadas al sistema político y al sistema de gobierno de cada Estado. (Mendoza, 2018, p. 64).

De acuerdo con Guillermo O’Donnell (1993), en América Latina existe un amplio sector de la población que padece lo que él denominó "ciudadanía de baja intensidad", donde los derechos civiles, políticos y culturales -en suma: lo derechos humanos universales- se ven socavados por las mismas instancias gubernamentales e instituciones políticas. Además, se puede caer en errores democráticos como contrarrestar los "problemas de pluralidad" con el exterminio, la creación de reservas o mediante políticas de asimilación o integración cultural (Brysk, 1995).

Así, la participación política indígena se concibe como el ejercicio de derechos tanto individuales como colectivos para influir en las decisiones en la esfera pública hacia el interior de la comunidad y en la obtención de representación política en el sistema político vigente, pues los derechos individuales de las personas indígenas tienden a materializarse cuando se ejercen en grupo (Henrard, 2005; Tomaselli, 2016). Además, de acuerdo con Ruiz Murrieta (2003) se pueden reconocer siete factores que favorecen la participación política indígena:

1) Progresiva transformación de las instituciones del Estado.

2) Creciente expansión territorial y demográfica de las personas y la cultura indígena hacia el interior de los estados. 
3) Fortalecimiento de las formas de organización indígena.

4) Desarrollo de mejores estrategias de lucha en materia jurídica y política por parte de las personas indígenas.

5) Inclusión de economías indígenas en la dinámica de mercado.

6) Creciente transformación de las relaciones entre los pueblos indígenas y el aparato estatal.

7) El surgimiento de herramientas jurídicas y políticas a nivel internacional.

Ahora bien, se tiene en consideración que la brecha de implementación (Martínez, 2012), entre lo que está estipulado en las leyes y la realidad sociopolítica, priva, por decirlo de alguna manera, de la capacidad real que tienen los pueblos y personas indígenas en México para ejercer ciudadanía. Además, la discriminación estructural en las grandes urbes mexicanas, la pobreza y la marginación a la que está supeditada la población indígena por el solo hecho de ser indígenas aumenta considerablemente las posibilidades de padecer una ciudadanía de baja intensidad (González Casanova, 2013; Hall \& Patrinos, 2005). Esto es importante, pues es propio de la ciencia política establecer que los recursos económicos de cada cual terminen transformándose en recursos políticos en determinado momento (Dahl, 1996). Pero qué sucede cuando 8,4 millones de personas indígenas viven en situación de pobreza en México, un número que representa casi el 70\% de la población indígena total en el país (CONEVAL, 2018).

En ese sentido, se puede entrever que la consulta indígena es un instrumento que tienen las personas y los pueblos indígenas para participar de manera efectiva en la toma de decisiones. No obstante, son diversos los actores involucrados en la materialización de las demandas de la población indígena. Asimismo, a continuación se dará un repaso breve de las características de la ZMM y cuáles han sido las principales características de los procesos de participación política de la población indígena en un contexto migratorio.

\subsection{A manera de contextualización}

Teniendo más de 350.000 personas que se autoidentifican como indígenas, en la zona metropolitana de Monterrey residen personas 
indígenas provenientes principalmente de San Luis Potosí, Veracruz, Estado de México, Oaxaca e Hidalgo -hablantes de náhuatl, huasteco, otomí, zapoteco, mixteco, tének, entre otras lenguas. Es conveniente señalar que la aparición de organizaciones de la sociedad civil indígenas y proindígenas ha tenido un papel importante en la protección de derechos indígenas, siendo Zihuakali, Zihuame Mochilla, RedMIN, Enlace Potosino, Procuración de Justicia Étnica y Proyecto Xipaki, algunas de las organizaciones más visibles (Wright \& Aguirre, 2018).

De acuerdo con la consultora neoyorkina Mercer (2017), la zona metropolitana de Monterrey ${ }^{7}$ (capital de Nuevo León) tiene la mejor calidad de vida en México. Empero, al mismo tiempo, fue considerada la ciudad más contaminada de México (Anguiano, 2018), con un déficit de árboles, un abrumante aumento de parque vehicular, exceso de empresas extractoras de caliza ${ }^{8}$, precaria calidad de infraestructura y movilidad en el transporte urbano, lo cual refleja desigualdades sociales (Apaolaza, Blanco, Lerena, López-Morales, Lukas \& Rivera, 2016). Además, en 2011, el Consejo Nacional para Prevenir la Discriminación (CONAPRED) -a través de la Encuesta Nacional sobre Discriminación en México- posicionó a la zona metropolitana de Monterrey como la zona urbana que más discrimina en México, siendo las principales causas de discriminación el origen étnico, estatus migratorio, preferencias sexuales y discapacidad.

Semejantes contrastes también se pueden observar en la vida social e institucional en Nuevo León. Por ejemplo, a partir de la década de los 90 se comienza a registrar en el país un aumento en la migración de personas indígenas hacia las grandes ciudades metropolitanas del país, como lo es Monterrey y su área metropolitana, trayendo consigo modificaciones en la vida social (Mendoza, 2019; Wright \& Aguirre, 2018) e institucional, como la creación en 1998 del Departamento de Educación Indígena (DEI) de la Secretaría de Educación de Nuevo

7 La zona metropolitana de Monterrey comprende 12 municipios y es la tercera ciudad más poblada de México, solo detrás de las áreas metropolitanas del Valle de México y Guadalajara.

8 También denominadas pedreras y una de las principales fuentes de contaminación con graves riesgos tanto para las áreas naturales protegidas como para la salud de la población. 
León y en 2012 la promulgación de la Ley de los Derechos Indígenas (Mendoza, 2019).

Si bien es cierto que los estudios en la entidad se han esforzado por conocer las particularidades culturales propias del proceso migratorio, los estudios sobre la "adaptabilidad estratégica" (Bartolomé, 2006) y/o formas de expresión políticas provocadas por los procesos migratorios (Cerda \& Gallegos, 2008; Durin, 2003) siguen siendo escasos como para conocer cómo participa políticamente la población indígena y su involucramiento con los diversos actores que, en principio, tendrían que promover su participación.

Por último, conviene destacar que en otras entidades federativas con zonas metropolitanas con características similares a las de la ZMM, como lo es Jalisco y la zona metropolitana de Guadalajara (ZMG), el papel de las OSC indígenas ha sido importante. Por ejemplo, estudios recientes como los de Mendoza, Martínez y Rodríguez (2020) señalan que la visibilización de la migración indígena en la ZMG y su incorporación a las instituciones de educación superior (Universidad de Guadalajara o el Instituto Tecnológico y de Estudios Superiores de Occidente) y a las del Estado (Consejo Estatal Indígena) ha sido motivado principalmente por OSC como Jóvenes Indígenas Urbanos (JIU) o Sueño de Mujeres Mixtecas, por mencionar algunas. En esta misma línea, Gracia y Horbath (2019) advierten que en la ZMG, grupos indígenas han desarrollado formas de cooperación que permiten contrarrestar las dificultades cotidianas como la discriminación al mismo tiempo que promueven y aprovechan las estructuras de oportunidades políticas (EOP) para el acercamiento con aliados estratégicos y con las instituciones públicas mismas.

\section{2 ¿Qué estipula la Ley de los Derechos Indígenas en Nuevo León?}

Publicada en junio de 2012, la Ley de Derechos Indígenas en Nuevo León tiene como objetivo valer la garantía, observancia, promoción y protección de los derechos y la cultura indígena, "y cuyos agentes procuradores son el Estado y los municipios de Nuevo León” (Mendoza, 2018, p. 107). En ese sentido, es preciso señalar que, por parte del Estado, es a través de la Secretaría de Desarrollo Social (SEDESOL), y de los municipios donde se concentra la mayor cantidad de personas in- 
dígenas ${ }^{9}$-en cualquiera de sus tres tipos de asentamientos estipulados por Durin (2003): aislado, congregado y disperso- son las administraciones locales las que tienen la obligación de garantizar la aplicación de lo establecido en la ley.

Es conveniente resaltar que, en términos sustantivos y para efectos del presente trabajo, la ley estipula principalmente lo siguiente: a) el artículo 8 brinda garantía de la libre determinación, autonomía y representación indígena para decidir formas de organización política; b) el artículo 9 establece el derecho de elegir representantes ante el ayuntamiento ${ }^{10}$ en función del respeto a sus usos y costumbres; y c) el artículo 28 estipula el derecho a la consulta en titularidad colectiva sobre las medidas y acciones que tomen los estados y/o municipios que les afecten de manera positiva o negativa en sus derechos colectivos y obligan a los estados y municipios a consultar a la población indígena en la elaboración del plan estatal y planes municipales de desarrollo. Como se puede observar, la Ley de los Derechos Indígenas en Nuevo León es clara en cuanto a las responsabilidades y acciones de cada orden o nivel de gobierno. Por ello, es conveniente destacar a continuación las acciones que ya se han implementado en Nuevo León en materia de participación indígena en la entidad.

\subsection{Procesos de participación indígena en Nuevo León}

Han sido variados los esfuerzos por parte de las autoridades gubernamentales en la promoción y cumplimiento de los derechos indígenas en la entidad. A continuación, se pasa revista de algunas acciones que se han realizado en la zona metropolitana de Monterrey:

1) En 1998 se crea el DEI -antes de la promulgación de la Ley.

2) En 2003 se realiza la apertura de la Oficina de Representación de la Comisión Nacional para el Desarrollo de los Pueblos Indígenas

9 Monterrey, García, Juárez, Escobedo, Apodaca y San Pedro Garza García, principalmente.

10 México es una República Federal y tiene 3 niveles de gobierno: federal, estatal y municipal. En este último, la composición política y administrativa es denominada Ayuntamiento. 
(CDI) -cuya delegación radicaba en San Luis Potosí ${ }^{11}$ - del gobierno federal, también antes de la promulgación de la Ley.

3) En 2011 se realiza el proceso de consulta por parte de la SEDESOL con apoyo de la CDI e instituciones de educación superior en el Estado y que, de acuerdo con Claire Wright y Néstor Aguirre (2018), "el proceso fue exitoso en el sentido que fomentó la participación de los actores interesados. No obstante, en términos de la calidad de la decisión tomada (...) es muy difícil establecer en qué medida los acuerdos se implementaron" (Wright \& Aguirre, 2018, pp. 194-195).

4) En 2016 se llevó a cabo la Consulta Nuevo León para el Plan Estatal de Desarrollo 2016-2021, donde, desde la nomenclatura hasta el proceso y resultado no quedó claro que fuera una consulta a la población indígena y más bien quedó en un plano simbólico y con pocos efectos reales y tangibles en materia de derechos indígenas para la población en Nuevo León (Wright \& Aguirre, 2018).

Tras lo anterior, ¿cuál ha sido el papel de las organizaciones civiles indígenas y proindígenas en estos procesos de participación política indígena? La siguiente sección pretenderá dar respuesta a esta pregunta de investigación a través del análisis de entrevistas semiestandarizadas realizadas a las principales asociaciones civiles en materia indígena en Nuevo León.

\section{El papel de las organizaciones civiles indígenas y proindígenas en la participación política de las personas indígenas}

Las irrupciones de los pueblos indígenas en la arena política convencional en América latina estuvieron íntimamente ligadas con las OSC y materializando lo que teóricamente Sidney Tarrow (2012) denominaba estructura de oportunidades políticas (Martí, 2010; Tarrow, 2012). De tal manera, se puede reconocer que la participación política indígena en México ha estado ampliamente relacionada con las OSC y los impactos se pueden analizar desde la vertiente constitucional, de au-

11 La CDI en 2018 pasa a llamarse Instituto Nacional de los Pueblos Indígenas (INPI) y la estructura organizacional cambia en forma y fondo, pues las delegaciones desaparecen y se reconfigura la descentralización de la dependencia. 
tonomía, del territorio y de vinculación con partidos políticos de corte indígena (Carbonell, 2003; González, 2010; Cott van, 1995).

Como se puede entrever, la vertiente de autonomía en términos del territorio o la vinculación con partidos políticos de corte indígena no se han materializado en Nuevo León debido a que no existen comunidades o pueblos originarios y, de los 51 municipios que conforman el Estado, ninguno llega a tener el $40 \%$ o más de población indígena ${ }^{12}$. De los municipios metropolitanos, es Escobedo y García los que mayor porcentaje de habitantes indígenas tiene, con $18,13 \%$ y $13,26 \%$, respectivamente; de los municipios no metropolitanos, destaca el municipio de Ciénega de Flores con 9.051 habitantes indígenas que representan el $21,19 \%$ y, además, es considerado uno de los municipios con mayor crecimiento anual en México (CEE, 2020; INEGI, 2015).

A pesar de lo anterior, en la zona metropolitana de Monterrey sí encontramos diversas organizaciones de la sociedad civil de carácter indígena -donde presidente y miembros de la organización son indígenas- y proindígenas -aquellas organizaciones donde no todos sus miembros son indígenas, pero sus trabajos son en beneficio de la población indígena- que tienen como finalidad la promoción y el respeto de derechos indígenas, como lo es el de participación política en la entidad (Mendoza, 2019).

Si bien es cierto que el actor responsable de la consulta indígena es el Estado, resulta de gran interés conocer de qué manera las OSC incentivan la participación política indígena o, en su defecto, obstaculizan la materialización de derechos indígenas. Es decir, se parte de la hipótesis que la proximidad a las OSC indígenas y proindígenas influye en la participación política indígena en Nuevo León. Es preciso señalar lo que Mendoza, Martínez y Rodríguez (2020) establecieron para el caso del ejercicio de ciudadanía de mujeres indígenas migrantes en la zona metropolitana de Monterrey y Guadalajara: "los grandes obstáculos a derribar en materia de ciudadanía de mujeres indígenas están construidos con base en el racismo, la discriminación y la exclusión

12 En México hay 623 municipios considerados indígenas, donde su población es de $40 \%$ o más habitantes indígenas y en su mayoría se encuentran en la zona sur y sureste del país. 
que cercenan los derechos, sus prácticas y su particularidad cultural" (p. 63).

\subsection{Método. ¿Cómo se realizó la aproximación empírica?}

Para estudiar el papel de las OSC indígenas y proindígenas en la materialización del derecho de participación política a través de la consulta indígena, se tuvo a bien tomar como punto de partida el enfoque de investigación cualitativa para analizar los procesos sociales y su impacto en las personas y comunidades. Además de cumplir con los preceptos básicos de la investigación cualitativa -teoría subjetiva, etnometodología y estructuralismo-, para el trabajo de campo se optó por la entrevista semiestandarizada, debido a que permite reconstruir la teoría subjetiva adherida al entrevistado en cuanto a sus conocimientos explícitos sobre el tema, articular preguntas basadas/guiadas en la teoría para hacer explícito lo implícito o rechazar los tópicos en función del bagaje cognitivo del entrevistado y finalizar con preguntas de confrontación que estén en oposición con las nociones previamente incrustadas por el entrevistado, para explorar la posibilidad de que tales nociones sean adheridas a la teoría subjetiva de este (Flick, 2007).

Con la finalidad de contestar la pregunta y comprobar la hipótesis de investigación, se realizaron 3 entrevistas semiestandarizadas a presidentes(as) de las OSC indígenas y proindígenas: Zihuame Mochilla, Zihuakali Casa de la Mujer Indígena y Nuevo León Árbol de Todas las Raíces con la pretensión de aproximarse al "mundo de ahí afuera y entender, describir y algunas veces explicar los fenómenos sociales desde el interior de varias maneras" (Flick, 2014, p. 12), como lo es el de la participación política indígena a través de las OSC y la consulta previa, libre e informada a personas indígenas.

Los datos recabados en las entrevistas semiestandarizadas fueron analizados a través del software NVivo12, lo que facilitó el tratamiento de datos de manera esquemática e inductiva a partir de dos categorías de análisis: participación política indígena y proximidad a OSC indígenas y proindígenas. Esto da espacio para la posibilidad de la emergencia de categorías analíticas desde la realidad a partir de la reconstrucción teórica en función de los puntos de vista y estructuración del problema mismo de parte de los informantes clave entrevistados (Flick, 2007). 


\subsection{Consulta indígena y OSC, ¿cumplimiento del derecho a la participación?}

"Pero pues ahí está la ley, pero tampoco se aplica". (Zihuakali Casa de la Mujer Indígena, 2018, entrevista).

Como se ha estipulado con anterioridad, la consulta indígena, además de ser un proceso y herramienta obligatoria para los tres niveles de gobierno en México, representa un mecanismo de participación política indígena y de obtención de representación política en las instancias correspondientes. Para el caso específico de Nuevo León, este proceso conlleva el involucramiento de OSC de carácter indígena y proindígena que promuevan y vinculen a la población indígena asentada en la entidad.

De esta manera, se pudo identificar que sí se cumplen los procesos y mecanismos de participación en términos formales de consulta indígena en 2011 para la promulgación de la Ley de los Derechos Indígenas en Nuevo León de 2012 y en 2016 para el Plan Estatal de Desarrollo. También se pudo reconocer que las OSC de la entidad son una vía asequible tanto para el Estado como para las personas indígenas al momento de participar:

Mira, la ley se promulga en el 2012, pero se trabajó durante 5 años con grupos de reflexión, con promesas de trabajo, o sea hubo como múltiples eh acontecimientos previos. (Zihuame Mochilla, 2018, entrevista).

Sin embargo, también se pueden reconocer discordancias en cuanto a si en verdad los acuerdos y peticiones en los procesos de consulta indígena resultan vinculantes, pues a la luz de la promulgación de la ley en 2012, los derechos estipulados en ella no se han visto materializados y ello permea en la manera cómo se da la evaluación de la consulta:

Nosotras como asociación civil somos invitadas especiales, pero no tenemos ni voz ni voto y luego la CDI, que es la Comisión Nacional para el Desarrollo de los Pueblos Indígenas, también está como observador, igual que la Comisión de Derechos Humanos, pero tampoco tienen ni voz ni voto, entonces como que en realidad no están. (Zihuakali Casa de la Mujer Indígena, 2018, entrevista). 
Lo anterior resulta importante, pues reafirma la "paradoja nodal" (Martínez, 2012) en cuanto participación y ejercicio de ciudadanúa indígena se trata. Es decir, existe una contradicción fundamental en el cumplimiento del derecho de participación política indígena debido a que son las mismas instituciones, los mismos actores y las mismas dinámicas que socavaron durante años y siglos los derechos de las personas y pueblos indígenas quienes ahora tienen el deber de cumplir y hacer valer sus derechos; un deber que no ha sido cumplido por las autoridades estatales, municipales, ni por parte de los representantes en el Congreso local.

Ante lo mostrado se establece que los peligros de que la ley se convierta en letra muerta o se instale dentro de la "brecha de implementación" son muy visibles y tangibles. Esto resulta alarmante, pues ya entrado el siglo XXI, la pugna deberá establecerse más allá del reconocimiento de derechos, para pasar al ejercicio y exigencia de estos:

No nada más un conocimiento de derechos, sino más allá, a exigirlos, a ejercerlos... pero emergen liderazgos muy fuertes y muy interesantes de analizar, ¿verdad? (Zihuame Mochilla, 2018, entrevista)

Sin embargo, para el caso de los derechos de participación indígena en Nuevo León, se sigue estando en la etapa de reconocimiento de derechos, pues, antes del ejercicio de derechos en función de las diferencias y diversidad cultural, se precisa de procesos multi e interculturales (Mendoza, Martínez \& Rodríguez, 2020). Por esta razón, la conformación de redes de apoyo es quizá el vínculo para pasar del reconocimiento al ejercicio de derechos de las personas indígenas en un contexto migratorio, como es el caso de la zona metropolitana de Monterrey.

El surgimiento de fuerzas exógenas como falsos liderazgos están presentes. Retomando la noción de la "doble dimensión de la participación indígena" de Alexandra Tomsalli (2016), podemos entrever que uno de los grandes retos que supone la articulación de la participación política indígena en Monterrey es que las dinámicas propias de la dimensión externa están altamente inmiscuidas en las dinámicas de la dimensión interna, es decir, los procesos de participación comunitaria propios de las comunidades de origen se van desdibujando en la zona metropolitana de Monterrey: 
Hace poco nos decían bueno hagan un focus group con los líderes comunitarios, no los puedes juntar porque están peleados... se ha perdido el sentido de comunidad, en la comunidad no es así, pero aquí... recuerdo mucho un evento de una chica que quiso hacer algo padre para la comunidad y empezó a gestionar, a pedir apoyo de que para alimento, para el sonido, para los payasos, era una posada en una comunidad, entonces recibió donaciones de juguetes, de distintos tipos de juguetes, y entonces convoca a la comunidad a pasar un rato agradable con los niños y demás y se hizo un caos, una pelea campal de que yo quería el mejor regalo para mi hijo y entonces te empujo, te piso y de la misma comunidad, salieron peleadas las vecinas. (Zihuakali Casa de la Mujer Indígena, 2018, entrevista).

Además, los conflictos entre asociaciones, liderazgos indígenas y las mismas instituciones gubernamentales podrían provocar un distanciamiento entre sí y con la población indígena en general:

Lo que se ha visto también en las leyes de consulta o en mesas de trabajo para propuestas políticas, hemos visto algunas otras asociaciones y compañeros que están en el activismo, algunos lideres comunitarios, pero son los mismos, más bien no tienen ubicados a bueno líderes, que están en pro de la comunidad, sino mas bien líderes que han aprendido de la política. (Zihuakali Casa de la Mujer Indígena, 2018, entrevista).

¿Qué quiere decir esto? Básicamente, la polítización de liderazgos indígenas podría significar una mayor muestra de desarrollo e institucionalización en las formas de participación política por parte de la población indígena, aunque también se corre el riesgo de polarizar y desincentivar la participación real, libre e informada de las personas indígenas:

Otras personas que, son puros varones entonces, ellos andan súper, súper politizados, mucho en la política, mucho, mucho, les gusta mucho y bueno andan en todo y ellos sí, mueven a la gente cuando tienen que recabar alguna firma o así con ellos... siempre llegan, nos preguntan nuestras necesidades, se llevan toda la información y luego ya ahí se acaba, o sea no hay más, no hay un seguimiento o qué van a hacer con esa información. (Zihuakali Casa de la Mujer Indígena, 2018, entrevista). 
Lo anterior demuestra que, si bien se puede cumplir con la consulta indígena, esto no representa que la población indígena en Nuevo León esté exenta de una suerte de clientelismo político por parte de "liderazgos" y OSC. A esto se le suma la constante tentación de que las instituciones gubernamentales incorporen a las OSC por motivos "folklóricos":

A Zihuame nos invitan mucho a diferentes mesas de trabajo, observatorios etc... en realidad en los que de verdad trabajamos y hay otros que perdón, pero pues nos invitan porque entonces dicen que incluyeron a la población indígena. (Zihuame Mochilla, 2018, entrevista).

Un último aspecto a considerar sobre la consulta indígena radica en dar cuenta de los alcances de la participación indígena para la obtención de representación política indígena como lo establece la ley. Es decir, a la luz de seis años de publicada la ley, tomando en cuenta la fecha de cuando se realizaron las entrevistas, se puede observar que el derecho de representación no queda garantizado:

Entrevistador: ¿Existen espacios de participación y representación indígena en el Estado?

Entrevistada: de representación, no.

(Zihuakali Casa de la Mujer Indígena, 2018, entrevista).

A pesar de lo anterior, se pudo reconocer que la perspectiva de que si hay o no representación indígena varía de una organización a otra y la variable de género como clave interpretativa es materia de profundo análisis, pues la OSC indígena cuyos integrantes son todos varones tienen mayor y mejor vínculo con las instituciones gubernamentales:

Entrevistado: en esa yo participé, pero participé en la que se convocó más con la organización indígena.

Entrevistador: ¿Hubo una para organización?

Entrevistado: Sí, en la Torre Administrativa, en esa pues nosotros todos saben, todos los que participaron sabía que el tema principal era tratar de posicionar que hubiera una dirección que atendiera y por eso quedó, el plan estatal no menciona de forma clara porque, pues, es general el plan estatal, pero el programa sectorial sí lo 
menciona y en ese sentido, en ese porque también nos volvieron a invitar también incluso a coordinar la mesa... en esas sí estuvieron investigadores, hubo mesas de investigadores, de representantes indígenas y en esa todas coincidieron y eso, pues, o sea sí hubo una incidencia o sea sí tuvo un resultado real que incidió, incidió en el plan estatal de desarrollo, o sea hay un apartado que habla de población indígena y eso, pues, es muy significativo.

(Nuevo León Árbol de Todas las Raíces, 2018, entrevista)

Cabe destacar que la confianza entre OSC indígenas y proindígenas está supeditada, pareciera, a la variable de género, pues a pesar de que estas OSC tienen como principales trabajos de intervención la prevención de la violencia contra la mujer y violencia familiar, la desconfianza entre OSC está muy marcada:

No nos hemos atrevido a participar tanto porque tenemos miedo de que nos vayan a utilizar, a que ellos hagan algo... porque ya lo han hecho... que nos utilicen como Asociación Civil... así que con mucha reserva, con mucha reserva, cuando nos hacen alguna invitación porque tienen esa doble intención. (Zihuakali Casa de la Mujer Indígena, 2018, entrevista).

En síntesis, la proximidad a OSC indígenas y proindígenas es de vital importancia para la participación política, empero, y a pesar de los resultados positivos de las consultas indígenas en el Estado, como lo fue la promulgación de la ley en 2012, los problemas de protagonismos de las propias OSC es una constante al momento de hablar de liderazgo e impacto real en la garantía de los derechos de las personas indígenas en Nuevo León. El trabajo en sinergia de las OSC indígenas y proindígenas se muestra aún incipiente y su consolidación estará supeditado a la apertura real de espacios de participación y de representación con mayor alcance.

\section{Reflexiones finales}

Se tuvo como finalidad conocer, explorar e interpretar de qué manera las organizaciones de la sociedad civil indígenas y proindígenas en Nuevo León se vinculan con los esfuerzos de incentivar la participación política indígena u obstaculizan la materialización de derechos indígenas. 
Tomando como mecanismo de participación política indígena a la consulta previa, libre e informada, se pretendió conocer si efectivamente la proximidad a las OSC indígenas y proindígenas influyen en la participación política. De manera concreta, se pudo reconocer que si bien han sido amplios los esfuerzos de las OSC antes mencionadas, persisten elementos en sus dinámicas que obstaculizan la participación política indígena en Nuevo León. Alguno de los principales obstáculos que se reconocieron fueron son los siguientes:

- Se carece de una figura por parte de las organizaciones de la sociedad civil indígenas o proindígenas que tenga voz y voto en el cumplimiento de demandas por parte de las instituciones gubernamentales.

- La paradoja nodal sigue presente en Nuevo León en el tema indígena, pues son las mismas instituciones, los mismos actores y las mismas dinámicas políticas que excluyeron a la población indígena quienes tienen ahora que cumplir, promover y respetar sus derechos.

- Las visiones y prácticas de "folclorización", tanto por parte de instituciones gubernamentales como de OSC proindígenas, siguen estigmatizando y estereotipando a la población indígena en Nuevo León, provocando con ello la reproducción de prácticas discriminatorias.

- Los falsos liderazgos indígenas promueven prácticas clientelares de corte político-electoral.

- El género sigue marcando la diferencia, pues son las OSC indígenas presididas por varones las que mayor contacto tienen con miembros de las instituciones gubernamentales y de otras instituciones políticas.

- La falta de sinergia y de trabajo en conjunto sigue obstaculizando la participación política, pues, además de no establecer agendas en conjunto, el alcance que se tiene con los miembros de la comunidad que son ajenos a las dinámicas políticas y a las organizaciones civiles resulta insuficiente para el cumplimiento de las demandas de la población indígena en general.

Usualmente existe la tendencia de concebir y analizar la participación política indígena desde la comunidad de origen. Sin embargo y 
como se ha demostrado, los procesos de participación política indígena se materializan más allá de sus comunidades de origen y territorios, y confluyen con diversas visiones de vida en ciudades de corte industrial, como es el caso de la zona metropolitana de Monterrey, en el Estado de Nuevo León en México.

El aumento de la migración al área metropolitana de Monterrey provocó la modificación de la forma en cómo respetar y hacer valer los derechos políticos indígenas consagrados en la CPEUM y en la Constitución local mediante la Ley de los Derechos Indígenas en Nuevo León. Si bien es cierto que ambas herramientas jurídico-políticas antes mencionadas promueven y brindan el carácter de obligatorio de la consulta indígena, se pudo reconocer que no basta con la implementación de la consulta indígena para garantizar de manera plena el derecho de participación y representación indígena en la entidad.

Si bien es cierto que trabajos de autores como Wright y Aguirre (2018), y Mendoza, Martínez y Rodríguez (2020) establecen que el reconocimiento por parte del Estado es el primer paso para el ejercicio de derechos indígenas en Nuevo León, en los resultados presentados con anterioridad se puede reconocer que actores como las OSC indígenas y proindígenas juegan un papel fundamental en el paso del reconocimiento al ejercicio de derechos. Además, la emergencia de la variable de confianza política resulta importante, pues si la desconfianza entre OSC persiste, la desconfianza de la población indígena a lo relacionado con el sistema político, sus instituciones y sus mecanismos democráticos, como la consulta previa, libre e informada, tenderá a aumentar.

La consulta previa, libre e informada es uno de los mecanismos que por ley están obligados los estados a implementar. Sin embargo y pesar de ser uno de los instrumentos democráticos de participación política con que cuenta la población indígena en Nuevo León, las demandas de las personas indígenas no han sido tomadas en cuenta de manera real, pues la discriminación sigue persistiendo en la entidad y el acceso a la educación, al trabajo digno o a la atención en materia de salud es precario. Además, desde 2012, año de publicación de la Ley de los Derechos de las Personas Indígenas y Afromexicanas en Nuevo León, la población indígena en la entidad no ha tenido representantes en el Congreso del Estado, los ayuntamientos no han incorporado las de- 
mandas indígenas a los planes municipales de desarrollo y en la academia el tema continúa siendo poco relevante.

Ir más allá de la resistencia y construir un diálogo entre las autoridades gubernamentales y las comunidades indígenas, en un contexto migratorio, encuentra cabida en la implementación del proceso de consulta indígena al momento de concebir un Estado intercultural para la construcción de una sociedad multicultural. No obstante, como se pudo demostrar, la participación política indígena en Monterrey y su área metropolitana, Nuevo León, por medio del proceso de consulta indígena, precisa de la participación activa de las OSC indígenas y proindígenas. En ese sentido, cabe destacar que se pueden reconocer aspectos que siguen obstaculizando la materialización de los derechos indígenas en la entidad y que son las OSC las que, en términos formales, sí cumplen con los procesos de participación, pero que en el fondo aún están lejos de considerarse como un vínculo efectivo que influya de manera real en la participación política indígena en la entidad, pues la falta de sinergia y desconfianza entre las OSC sigue siendo un obstáculo para la participación indígena a través de la consulta previa.

\section{Referencias}

Anguiano, D. (2018, febrero). Monterrey, la ciudad más contaminada [grupo de noticias]. El Financiero. Recuperado de https:// www.elfinanciero.com.mx/monterrey/la-ciudad-mas-contaminada

Apaolaza, R., Blanco, J., Lerena, N., López-Morales, E., Lukas, M. \& Rivera, M. (2016). Transporte, desigualdad social y capital espacial: análisis comparativo entre Buenos Aires y Santiago de Chile. Íconos. Revista de Ciencias Sociales, (56), 19-41. doi: 10.17141/iconos.56.2016.2148

Assies, W. (2009). Pueblos indígenas y sus demandas en los sistemas políticos. Revista CIDOB d'Afers Internacionals, (85/86), 89107. Recuperado de https://www.jstor.org/stable/40586388

Bartolomé, M. (2006). Procesos interculturales. Antropología política del pluralismo cultural en América Latina.México, D.F.: Siglo XXI.

Brysk, A. (1995). Acting globally: indian rights and international politics in Latin America. En D. van Cott (Ed.), Indigenous peoples 
and democracy in Latin America (pp. 29-51). Nueva York: St. Martin`s Press.

Cairo, C. \& Rozo, E. (2006). Políticas de la identidad, ciudadanía intercultural y reivindicaciones territoriales indígenas en dos localidades amazónicas. Universitas humanísticas, 61, 107134. Recuperado de https://revistas.javeriana.edu.co/index. php/univhumanistica/article/view/2081

Carbonell, M. (2003). La reforma constitucional en materia indígena. Un primer acercamiento. En Tribunal Electoral de la Federación (Ed.), Derechos indígenas y elecciones (pp. 49-70). México, D.F.: Tribunal Electoral del Poder Judicial de la Federación.

Cerda, J. \& Gallegos, L. (2008). La migración indígena hacia el noreste de México: Oportunidades y retos de la acción institucional en el caso del Estado de Nuevo León. Monterrey: Comisión Nacional para el Desarrollo de los Pueblos Indígenas.

Cohen, J. \& Arato, A. (1992). Civil society and political theory. Cambridge: MIT.

Comisión Estatal Electoral de Nuevo León. (2020). Acuerdo CEECG-59-2020. Recuperado de https://www.ceenl.mx/consulta/2020/consulta_indigena/misc/docs/Acuerdo\%20CEECG-59-2020.pdf

Consejo Nacional de Evaluación de la Política de Desarrollo Social (2018). Informe de Evaluación de la Política de Desarrollo Social 2018. México, D.F.: CONEVAL.

Cott van, D. (1995). Indigenous peoples and democracy: issues for policymakers. En D. van Cott (Ed.), Indigenous peoples and democracy in Latin America (pp. 1-28). Nueva York: St. Martin`s Press.

Dahl, R. (1996). Equality versus inequality. PS: Political Science \& Politics, 29(4), 639-648. doi: 10.1017/S1049096500045601

Domínguez, A. \& Santiago, G. (2014). Un acercamiento a la participación político electoral de los pueblos indígenas en México. Revista Latinoamericana de Estudios Educativos, 44(3), 83-136. Recuperado de https://www.redalyc.org/ pdf/270/27032150005.pdf

Durin, S. (2003). Nuevo León, un nuevo destino de la migración indígena. Revista de Antropología Experimental, (3), 1-7. Recuperado de https://revistaselectronicas.ujaen.es/index.php/ $\mathrm{rae} /$ article/view/2103 
Flick, U. (2007). Introducción a la investigación cualitativa. Madrid: Morata.

Flick, U. (2014). La gestión de la calidad en investigación cualitativa. Madrid: Morata.

García, D. (2013). La espacialidad de los indígenas en el área metropolitana de Monterrey. Relaciones, 134, 57-92. Recuperado de http://www.scielo.org.mx/pdf/rz/v34n134/v34n134a3.pdf

González, J. (2010). El Estado, los indígenas y el derecho. México, D.F.: Universidad Nacional Autónoma de México.

González Casanova, P. (2013). La democracia en México. México, D.F.: Era.

Gracia, M. \& Horbath, J. (2019). Exclusión y discriminación de indígenas en Guadalajara, México. Perfiles Latinoamericanos, 27(53), 1-24. doi: 10.18504/pl2753-011-2019

Granados, J. (2005). Las nuevas zonas de atracción de migrantes indígenas en México. Investigaciones Geográficas, 58, 140-147. doi: 10.14350/rig.30052

Hall, G. \& Patrinos, A. (2005). Indigenous Peoples, Poverty and Human Development in Latin America. Londres: Palgrave Macmillan.

Henrard, K. (2005). "Participation", “representation”, and "autonomy" in the Lund recommendations and their reflections in the supervision of the FCNM and Several human rights conventions. International Journal on Minority and Group Rights, 12(2-3), 133-168. doi: 10.1163/157181105774740606

INEGI. (2015). Encuesta Intercensal. Panorama sociodemográfico de Nuevo León . Nuevo León: INEGI. Recuperado de https://www.inegi.org.mx/app/biblioteca/ficha. html?upc $=702825082291$

Juan-Martínez, V. (2019). Fiscalizando la autonomía. Estado, pueblos indígenas y rendición de cuentas. Íconos. Revista de Ciencias Sociales, 65(23), 115-134. doi: 10.17141/iconos.65.2019.3799

Keck, M. \& Sikkink, K. (1998). Activist beyond borders: advocacy networks in international politics. Ithaca, Nueva York: Cornel University Press.

Kymlicka, W. (1996). Ciudadanía multicultural. Barcelona: Paidós.

López, F. (2006). Autonomía y derechos indígenas en México. México, D.F.: Ce-Acatl, A.C. 
Marañón, F., Saldierna, A., \& Muñiz, C. (2013). La presencia indígena en los programas de ficción y entretenimiento de la televisión mexicana. En C. Muñiz (Ed.), Medios de comunicación y prejuicio hacia los indígenas (pp. 51-66). México, D.F.: Fontamara.

Martí, S. (2010). The emergence of indigenous movements in Latin America and their impact on the Latin American political scene. Latin American Perspectives 37 (6), 74-92. doi: 10.1177/0094582X10382100

Martí, S. (2013). Balance y retos de la lucha de las poblaciones indígenas en el cambio de ciclo. En S. Martí, C. Wright, J. Aylwin \& N. Yáñez (Eds.), Entre el desarrollo y el buen vivir (pp. 21-42). Madrid: La Catarata.

Martínez, M. (2012). La paradoja irresuelta. Apuntes para el estudio de los derechos y la ciudadanía de los pueblos indígenas en México. Elecciones, 11(12), 235-263. Recuperado de https://www.academia.edu/27422385/La_paradoja_irresuelta_Apuntes_para_el_estudio_de_los_derechos_y_la_ ciudadan\%C3\%ADa_de_los_pueblos_ind\%C3\%ADgenas_ en_M\%C3\%A9xico

Martínez, M. (2015). Reconocimiento sin implementación. Un balance sobre los derechos de los pueblos indígenas en América Latina. Revista Mexicana de Ciencias Politicas y Sociales, 60(224), 251-278. doi: 10.1016/S0185-1918(15)30010-6

Melgar, R. (1991). Las categorías utópicas de la resistencia étnica en América Latina. Cuilco, 27, 49-60

Mendoza, F. (2018). Participación político-electoral indígena como elemento axial del sistema político. Una revisión teórica. En Wright, C. (coord.), Participación política indígena en México. Experiencias de gestión comunitaria, participación institucional y consulta previa (pp. 63-96). México, D.F.: ITACA.

Mendoza, J. (2019). Participación político-electoral de personas indígenas en ciudades industriales. Análisis de Monterrey y su área metropolitana. (Tesis doctoral inédita). Universidad Autónoma de Nuevo León, México.

Mendoza, J., Martínez, Z. \& Rodríguez, R. (2020). La construcción de ciudadanía en mujeres indígenas migrantes de las zonas metropolitanas de Monterrey y Guadalajara. iContinuidad o reconfiguración sociopolítica? Encuentros. Revista de Cien- 
cias Humanas, Teoría Social y Pensamiento Crítico, (12), 55-74. Recuperado de http://encuentros.unermb.web.ve/ index.php/encuentros/article/view/112

Mercer. (2017). Quality of living city rankings. Nueva York: Mercer. Recuperado de: https://www.mercer.com/newsroom/2017quality-of-living-survey.html

Monsiváis, C. (2014). México a principios del siglo XXI: la globalización, el determinismo, la nación ampliada y globalizada. En E. Subirats (Ed.), América Latina y la guerra global (pp. 2756). México, D.F.: Fondo de Cultura Económica.

Muñoz, H. (2014). En pos del derecho con mandato y recursos: una consulta sobre educación a comunidades indígenas de México. En A. Tomaselli, S. Ordóñez \& C. Wright (Eds.), Justicia y formas de participación indígena (pp. 75-103). Bilbao: Universidad de Deusto.

Naciones Unidas. (2007). Declaración de Naciones Unidas sobre los derechos de los pueblos indígenas. Ginebra: Naciones Unidas. Recuperado de https://www.un.org/esa/socdev/unpfii/documents/DRIPS_es.pdf

Obieta Chalbaud, J. (1993). El Derecho humano de la autodeterminación de los pueblos. Madrid: Tecnos.

Ochman, M. (2006). La reconfiguración de la ciudadanía. Los retos del globalismo y de la posmodernidad. México, D.F.: Instituto Tecnológico y de Estudios Superiores de Monterrey, Porrúa.

O’Donnell, G. (1993). Estado, democratización y ciudadanía. Nueva Sociedad, (128), 62-87. Recuperado de https://nuso.org/articulo/estado-democratizacion-y-ciudadania/

Organización Internacional del Trabajo, OIT. (2014). Convenio Núm. 169 sobre los pueblos indígenas y tribales en países independientes. Lima: Organización Internacional del Trabajo, Oficina Regional para América Latina y el Caribe. Recuperado de https://www.ilo.org/dyn/normlex/es/f?p=NORMLEXPUB:1 2100:0::NO::P12100_INSTRUMENT_ID:312314

Pajuelo, R. (2006). Participación pdolítica indígena en la sierra peruana. Una aproximación desde las dinámicas nacionales y locales. Lima: Instituto de Estudios Peruanos, Fundación Konrad Adenauer.

Rea Granados, S. (2015). Derecho a la consulta y la participación de los pueblos indígenas, la experiencia constitucional en los casos 
de México y Chile. Boletín Mexicano de Derecho Comparado, (144), 1083-1117. doi: 10.22201/iij.24484873e.2015.144.4961

Ruíz Murrieta, J. (2003). Democracia y participación política de los pueblos indígenas en América Latina. Gestión de las Transformaciones Sociales (MOST), Documentos de debate, 67. París: UNESCO.

Tamayo, S. (2006). Entre la ciudadanía diferenciada y la ciudadanía indígena ¿otra es posible? El Cotidiano, 21(137): 7-17. Recuperado de https://www.redalyc.org/pdf/325/32513703.pdf

Tarrow, S. (2012). El poder en movimiento. Los movimientos sociales, la acción colectiva y la política. Madrid: Alianza.

Tomaselli, A. (2016). Indigenous people and their right to political participation: international law standards and their application in Latin America. Baden-Baden, Alemania: Nomos.

Tresierra, J. (1995). Mexico: indigenous people and the Nation-State. En D. van Cott (Ed.), Indigenous peoples and democracy in Latin America (pp. 187-210). Nueva York: St. Martin`s Press.

Wright, C. (2018). El derecho a la consulta de los pueblos indígenas de México. Un balance de su reconocimiento, implementación e instrumentalización. En C. Wright (Ed.), Participación política indígena en México. Experiencias de gestión comunitaria, participación institucional y consulta previa (pp. 219256). Monterrey, México: Ítaca.

Wright, C. \& Aguirre, N. (2018). La consulta previa como herramienta de la multiculturalidad en contextos migratorios: evidencia desde Nuevo León, México. Revista Chilena de Derecho y Ciencia Política, 9(2), 178-201. Recuperado de http:// derechoycienciapolitica.uct.cl/index.php/RDCP/article/ view/1787

\section{Entrevistas}

Entrevista a Presidenta de Zihuakali Casa de la Mujer Indígena, centro de Monterrey, 5 junio de 2018.

Entrevista a Presidenta de Zihuame Mochilla, centro de Monterrey, 10 de junio de 2018.

Entrevista a Presidente de Nuevo León Árbol de Todas las Raíces, centro de Monterrey, 12 de junio de 2018. 